\title{
Clinical and Laboratory Profile of Children with Eosinophilia at Dhulikhel Hospital
}

\author{
Shrestha S, Dongol Singh S, Shrestha NC, Shrestha RPB
}

Deparment of Pediatrics,

Dhulikhel Hospital, Kathmandu University Hospital

Kathmandu University School of Medical Sciences

Dhulikhel, Nepal

Corresponding Author

Shrima Shrestha

Department of Pediatrics

Dhulikbel Hospital, Kathmandu University Hospital

Kathmandu University School of Medical Sciences

Dhulikhel, Nepal

E-Mail: sima_shrestha@yahoo.com.

\section{Citation}

Shrestha S, Dongol Singh S, Shrestha NC, Shrestha RPB . Clinical and Laboratory Profile of Children with Eosinophilia at Dhulikhel Hospital. Kathmandu Univ Med J 2012;38(2):58-62.

\begin{abstract}
Background

Eosinophilia in children is commonly caused by or parasitic, allergic and immunologic problems.
\end{abstract}

\section{Objectives}

To study clinical and laboratory profile of patients with eosinophilia and to identify possible causes.

\section{Method}

A prospective and descriptive study was conducted from January 2009- December 2011. All the cases of eosinophilia with symptoms and signs of single or multiple organ were included. The known caused of eosinophilia like allergy, asthma and drugs were excluded.

\section{Results}

During three years period, $84(2.41 \%)$ cases had eosinophilia with single or multiple organ involment. $14.3 \%, 39.3 \%$ and $46.4 \%$ had mild, moderate and severe eosinophilia respectively, with cases of hypereosinophilia comprising $85.7 \%$. Eosinophilia is seen predominantly in tamang caste with overall age ranging from 1-14 years. Most common symptoms and signs are abdominal pain (67.9\%) hepatomegaly $(59.5 \%)$ respectively. Gastrointestinal system was most commonly involved organ followed by respiratory system. Nineteen percent had polyserositis involving pleural, pericardial effusion and ascites at presentation. Out of 84 patients only nine serum samples were able to be sent for parasitological analysis. sixteen had identifiable and/ or possible causes. Serum sample for parasitological analysis revealed fascilosis, filariasis, strongylosis stercoralis and toxocariasis.

\section{Conclusion}

Eosinophilia is more common among tamang poulation in our study. Most common symptoms and signs are abdominal pain and hepatomegaly respectively. Parasitic infection seems to be the most common cause however further study has to be done to reach final conclusion.

\section{KEY WORDS}

Children, eosinophilia

\section{INTRODUCTION}

Parasitic infections are the most common cause of eosinophilia worldwide, although eosinophilia in the population of the United States is most often due to allergies. ${ }^{1}$ Less common causes of eosinophilia include some unusual pulmonary syndromes, such as Loeffler's syndrome (fibroplastic endocarditis), chronic eosinophilic pneumonia, reactive eosinophilia, and some autoimmune diseases such as rheumatoid arthritis, inflammatory bowel disease, and lupus erythematosus. ${ }^{2}$ Malignancies can also cause eosinophilia; examples are some of the leukemias, lymphomas, plasma cell dyscrasias, myelodysplastic disorders, and cancers of the lung, colon, pancreas, or cervix., With autoimmune diseases like rheumatoid arthritis, Crohn's disease, ulcerative colitis, and eosinophilic gastroenteritis, eosinophilia tends to occur primarily in patients with long-standing disease. ${ }^{3}$

Persistent significant eosinophilia should be regarded pathological and detailed investigations should be carried out to uncover a treatable cause. Mild or moderate increase in blood eosinophil counts detected from differential leucocyte counts may be encountered during routine health screening as an isolated laboratory abnormality without an apparent association with the disease or as an 


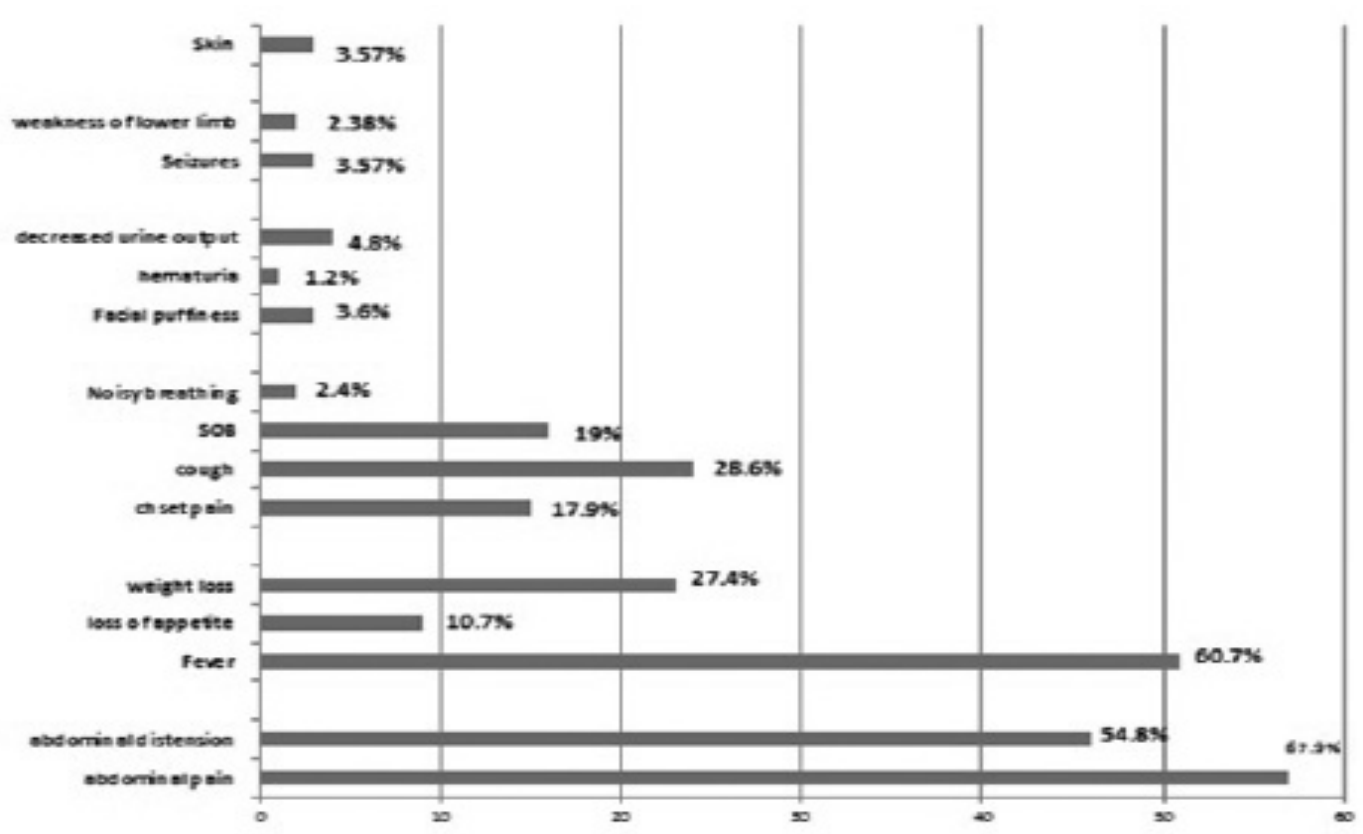

Figure 1. Symptoms in patient with eosinophilia.

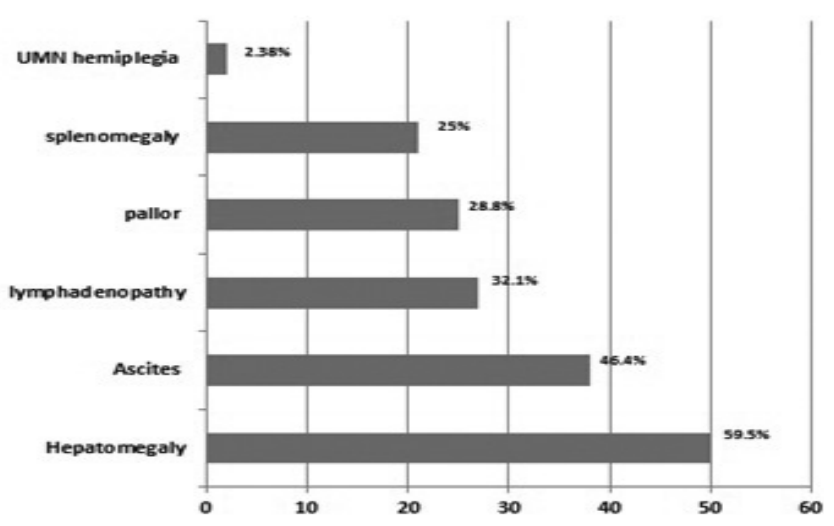

Figure 2. Signs in patient with eosinophilia.

epiphenomenon during a diagnostic work up for an illness. Normal eosinophil count in the human blood varies from $0-350 / \mathrm{mm} 3$ of the blood. ${ }^{5}$ This amounts to about $1-3 \%$ of the differential leukocyte count. According to Marc.E. Rothenberg classification eosinophilia is divided into mild: $351-1500 / \mathrm{mm} 3$ of blood, moderate: $1500-5000 / \mathrm{mm} 3$ of blood, severe: $>5000 / \mathrm{mm} 3$ of blood. ${ }^{5}$ The aim of the study was to study clinical and laboratory profile of patients with Teosinophilia and to identify possible causes.

\section{METHODS}

A prospective discriptive study was conducted from January 2009 to December 2011, at Dhulikhel hospital, Kathmandu University Hospital. Approval from the Institution and parents were taken. All the children with eosinophilia with organ involvement who presented in outpatient department or admitted in ward were included. Children with eosinophilia due to drug reaction, known allergy and asthma were excluded. Detailed clinical history and abnormal physical findings were recorded. Absolute eosinophilic count $>350 /$ microlitre was regarded as absolute eosinophilia. It is divideded into mild, moderate and severe according to Marc. E. Rothenberg classification. ${ }^{5}$

Routine investigations included complete blood count with peripheral smear examination, erythrocyte sedimentation rate (ESR), chest $X$ ray and consecutive three stool samples for parasites, ova and cysts.

Serum sample were stored at -20 degree celcius for serological analysis for parasites which was sent abroad whenever feasible. Depending on symptoms and signs of organ involvement, liver function test, echocardiography, and ultrasonpogram abdomen, pleural fluid, pericardial and asctic fluid analysis were done. HIV (human immunodefficiency virus) test, bone marrow examination, IgE (Immunoglobulin level E) level was done wherever possible.

\section{RESULTS}

Out of 3486 cases admitted in ward, 84 cases had persistent eosinophilia with organ involvement comprising incident of $2.41 \%$. Tamang / Lama/ Magar comprised of 57 (67.9\%) in which Tamang population was 29 (34.5\%) followed by Kami / Thami 14 (16.7\%), Newar 7 (8\%) and Brhamin/Chhetri $6(7 \%)$. Children from the rural area had higher incidence 73 (86.9\%). Though Tamang population and rural area had Table 1. System involvement in patient with eosinophilia.

\begin{tabular}{ll} 
System involvement & No. of cases (\%) \\
\hline Gastrointestinal & $57(67.9)$ \\
\hline Respiratory & $54(64.3)$ \\
\hline Cardiac & $28(33.3)$ \\
\hline Multisystem & $16(19)$ \\
\hline System involvement & $4(4.76)$ \\
\hline Musculoskeletal & $3(3.57)$ \\
\hline Renal & $1(1.2)$
\end{tabular}


maximum incidence its relation to mild, moderate and severe eosinophlia was statistically not significant with $\mathrm{p}$ value $>0.05$. The $\mathrm{RR}$ (relative risk) $(95 \% \mathrm{Cl})$ (confidence interval) of Tamang population for severe eosinophilia was 2.2(0.88-5.58). In our study group children belonging to $5-10$ years had maximum frequency of $40.5 \%$ with age range of 1-13 year with mean \pm S.D (standard deviation) of 7.4 year \pm 3.65 . Mean duration of symptoms was 55 days. Male to female ratio was 2.1:1.

The most common symptoms were abdominal pain 57 (67.9\%) followed by fever 51(60.7\%) and abdominal distension 46 (54.8\%). Respiratory symptoms include cough 24 (28.6\%), chest pain 15 (17.9\%). Three (3.6\%)children presented with facial puffiness and joint pain and swelling. Two cases $(2.4 \%)$ presented with seizure and hemiplegia. (fig 1)

The most common sign was hepatomegaly in 50 (59.51\%), ascites 38 (45.2\%), lymphadenopathy 27 (32.1\%).Most common organ involvement was gasstrointestinal tract followed by respiratory system comprising $64.3 \%$ and $67.9 \%$ respectively. (fig 2) System incolvement are shown in table 1 . However 16 (19\%) had multiple system involvement including systemic features, respiratory, cardiovascular and gastrointestinal system at the time of presentation. Regarding severity 12 (14.3\%), 33 (39.3\%), 39 (46.4\%) had mild, moderate and severe eosinophilia respectively.

The minimum and maximum differential eosinophil count at the time of entry of subjects into the study was $5-85 \%$. The minimum and maximum absolute eosinophil count was 605 and 32,850 respectively with mean of 9263.42 . ESR was normal in 53(63.1\%).Stool routine examination revealed normal in 74(88.1\%), cyst of Giardia lamblia $5(6 \%)$, Ova of Ascariasis in 3 (3.6\%), Cyst of E. histolytica in $1(1.2 \%)$ and Ova of Tricuris in 1 (1.2\%). Ultrasonography abdomen revealed normal findings in $12(24 \%)$, echogenic lesion in liverin $3(6 \%)$ and ascites with hepatomegaly in $35(70 \%)$.

\section{Table 2. Chest X Ray findings.}

\begin{tabular}{|ll|}
\hline CXR findings & No. of cases (\%) \\
\hline Normal & $40(48)$ \\
\hline Unilateral pleural effusion & $19(23)$ \\
\hline Effusion with cardiomegaly & $12(14)$ \\
\hline Bilateral pleural effusion & $5(6)$ \\
\hline Bilateral pulmonary infiltrates & $5(6)$ \\
\hline Only cardiomegaly & $3(3)$ \\
\hline
\end{tabular}

CXR results are shown in table 2. Pleural fluid analysis done in 19 (22.6\%) in which all were exudative and significant pleural eosinophilia seen in nine $(10.7 \%)$ cases. Pleural fluid gram stain was negative in 11 cases and two cases revealed pus cells.Pericardial fluid analysis was done in fifteen cases (17.86\%). Ten (12\%) presented with pericardial tamponade requiring pericardiocentesis.Ascitic fluid analysis done in 12 cases (14.3\%). In all the fluid analysis revealed exudate in nature however AFB and gram stain were negative.

Mantoux was positive in five $(5.95 \%)$ only.Bone marrow done in only fifteen (17.86\%) cases and revealed myeloid hyperplasia with eosinophilia in all cases. HIV test in eight (11.9\%) which were negative. ANA (anti nuclear antibody titre) in three cases were negative. Rheumatoid factor in three (3.57\%) and all negative. In four cases Ig E level were done and all were raised.Out of 84 cases serological analysis could be sent only in eight cases. Definitive diagnosis was made in $20(23.8 \%)$ cases shown in table 3.The probable tropical pulmonary eosinophilia in $5(5.95 \%)$ cases and diagnosis unknown in 59(70.24\%).

Table 3. Definitive diagnosis in patient with eosinophilia.

\begin{tabular}{|l|l|}
\hline Definitive diagnosis & $2(2.38)$ \\
\hline Strongyloidosis & $1(1.19)$ \\
\hline Filariasis & $1(1.19)$ \\
\hline Fasciolosis & $1(1.19)$ \\
\hline Strongyloidosis + Filariasis & \\
\hline+ Fasciolosis & $1(1.19)$ \\
\hline Toxocariasis & $1(1.19)$ \\
\hline Fasciolosis + Toxocariasis & $1(1.19)$ \\
\hline Hypereosinophilic & \\
\hline syndrome & $5(5.95)$ \\
\hline Ascariasis & $1(1.19$ \\
\hline Trichuris Trichuria & $1(1.19$ \\
\hline Tubercular Meningitis & $4(4.76)$ \\
\hline Disseminated TB & $1(1.19)$ \\
\hline Pilocytic Astrocytoma & \\
\hline
\end{tabular}

\section{DISCUSSION}

Eosinophilia can arise from an extensive number of medical conditions, including allergic disorders, hematologic, and other neoplastic diseases and infections, particularly helminthic. ${ }^{5}$

The differential diagnosis of eosinophilia is extensive. In industrialized countries, multiple medical conditions need to be considered, among them a variety of malignant, atopic, inflammatory, and endocrine conditions. ${ }^{5,6}$ However, in developing countries, infectious diseases account for the majority of cases of eosinophilia. ${ }^{7}$ By far, the infectious diseases with the strongest association with eosinophilia are helminthic. Other infectious disorders may be associated with eosinophilia (including Coccidioides, Aspergillus, Isospora belli, Dientamoeba fragilis, Mycobacterium leprae, chronic Mycobacterium tuberculosis, although the data supporting the association in some cases is inconclusive. For example, in developing countries, many patients with tuberculosis or Isosporiasis and eosinophilia are actually found to be co-infected with helminthes. ${ }^{8,9}$ In our case also one patient found to be co infected with tuberculosis and toxocariasis. The diagnosis of eosinophilia in tropical countries presents a difficult problem as parasitic infections are extremely common 
among other conditions causing eosinophilia. In contrast, allergy, skin disease, drug reactions, leukemia, malignant reticuloses and collagen disorders are the usual causes of eosinophilia in temperate zone, where parasitic infection is uncommon. However as dogs and cats are common domestic pets and children are continuously exposed to the hazards of animal nematodes, Toxocara infection is not an uncommon cause of eosinophilia in children in nontropical countries.

Usually patients of eosinophilia are asymptomatic as reported in previous study..$^{10}$ They were generally detected incidentally during routine hematological investigation. However In our study, most of the patients were symptomatic similar to study conducted by Makkar et al in Lady Harding Medical College. ${ }^{11}$

Atopy and parasitism are two important causes of eosinophilia. But etiology remains idiopathic in most patient. ${ }^{10,12}$ Allergy and atopy is described as the leading cause in developed countries and parasitism in travellers returning from the developing countries..$^{6,13,14}$

Similar study in Lady Harding Medical College revealed $52 \%, 34 \%$ and $14 \%$ cases with mild, moderate and severe eosinophilia respectively. ${ }^{11}$ However in our study $46.4 \%$ belong to severe category suggesting severe nature of the disease in a given cohort in our study.

Similar to the finding by Makkar et al where common symptoms were anorexia (40\%), pain in abdomen $38 \%$, fever $32 \%$ and breathlessness $30 \%$. In our study most common symptoms were abdominal pain (67.9\%) followed by fever and abdominal distension. In the same study serological test were not done, however $16 \%$ cases stool for ova and cyst were positive but in our study $26 \%$ had positive results. Similar to our finding bone marrow examination didn't revealed any diagnostic clue however in both the study clonal analysis were not done which was the limitation of our study. Study by Makkar et al showed two patients had tuberculosis but in our study definite evidence of tuberculosis was seen in three cases (3.6\%). ${ }^{11}$ Hence from both the study we can conclude tuberculosis in uncommon cause in a patient with eosinophilia. In our study two cases had Toxocara who presented with cardiac tamponade at the presentation. Baldisserotte et al described 18 cases of toxocariasis with hepatomegaly $(72.7 \%)$, splenomegaly (50\%), cervical lymphadenitis (33.3\%), pulmonary symptoms $(27.2 \%)$ and fever $(22.2 \%)$ but none had presented with cardiac tamponade. ${ }^{15}$ Henry et al reported 50 years man with toxocariasis with

\section{REFERENCES}

1. Holland SM, Gallin JI. Disorders of granulocytes and monocytes. In: Braunwald E, Fauci AS, Kasper DL eds. Harrison's Principles of Internal Medicine. 15th ed.New York, NY: McGraw-Hill; 2001.

2. Tefferi A. Blood eosinophilia: a new paradigm in disease classification, diagnosis, and treatment. Mayo Clin Proc. 2005 Jan;80(1):75-83.

3. Brigden ML. A practical workup for eosinophilia. Postgrad Med. 1999 Mar;105(3):193-4, 199-202, 207-10. cardiac tamponade. Yoko et al in Japan had reported in a 13 years girl with toxocariasis presenting with cardiac tamponade. ${ }^{16,17}$

Eosinophilic meningitis is rare in Western countries, but is sometimes seen in association with tuberculosis, syphilis, and coccidioidomycosis.$^{18}$ The disease is more common in South East Asia and Pacific regions. Angiostrongyloides cantonensis is the most frequent etiologic agent in these regions. Other helminthic infections that can cause eosinophilic meningitis include Cysticercosis, Schistosomiasis, Paragonimiasis, and Echinococcosis. Gnanthostoma spinigerum causes a particularly severe form of eosinophilic myeloencephalitis that may result in nerve root pain, paralysis of an extremity, and subarachnoid hemorrhage associated with visceral and cutaneous larvae migrans.$^{19}$

In our study three cases of eosinophilic meningitis have been encountered. the cause were filiariasis, fasciolosis and tubercular meningitis. Two cases ( 8 \& $10 \mathrm{yrs}$ ) have been reported as Fasciolosis with Brain haemorrhage by Ying $M$ et al and Zhou $L$ et al respectively. ${ }^{20,21}$.

Study in Korea revealed $12.2 \%$ and $0.9 \%$ were found to have eosinophilia and hypereosinophilia, respectively. ${ }^{22}$ Among patients with hypereosinophilia, $60.9 \%$ had identifiable and/or possible causes. The major causes of hypereosinophilia were malignancy (35.2\%), allergy and skin diseases (18.1\%), infectious diseases (15.4\%), hepatobiliary diseases (7.5\%), bone marrow clonal diseases (6.6\%) and parasite infections (6.6\%). They also found a rare case of FIP1L1-PDGFR alpha positive chronic eosinophilic leukemia combined with light chain multiple myeloma.

The limitation of our study was serological analysiscould not be performed in all cases, human immunodefficiency virus test and bone marrow examination could not be done in patients with multisystem involment.

\section{CONCLUSION}

Significant proportion of the population with eosinophilia belong to Tamang caste. Gastrointestinal system were the most common organ involvment followed by respiratory system in patient with eosinophilia. Tuberculosis is less likely cause of eosinophilia. Though serological analysis could not be done, parasitic infections are most common possible cause of eosinophilia. Serological analysis should be performed to reach the final conclusion regarding etiology.

4. Spiers AS. Management of the chronic leukemias: special considerations in the elderly patient, Part III rarer chronic myeloid leukemias. Hematology. 2002 Feb;7(1):1-8.

5. Rothenberg ME. Eosinophilia. N Engl J Med. 1998 May 28;338(22):1592-600. 
6. Brigden M, Graydon C, 1997. Eosinophilia detected by automated blood cell counting in ambulatory North American outpatients. Incidence and clinical significance. Arch Pathol Lab Med. 1997 Sep;121(9):963-7.

7. Nutman TB, Ottesen EA, leng S, Samuels J, Kimball E, Lutkoski M et.al. Eosinophilia in Southeast Asian refugees: evaluation at a referral center. J Infect Dis. 1987 Feb;155(2):309-13.

8. Certad G, Arenas-Pinto A, Pocaterra L, Ferrara G, Castro J, Bello A et.al. Isosporiasis in Venezuelan adults infected with human immunodeficiency virus: clinical characterization. Am J Trop Med Hyg. 2003 Aug;69(2):217-22.

9. Tristão-Sá R, Ribeiro-Rodrigues R, Johnson LT, Pereira FE, Dietze R. Intestinal nematodes and pulmonary tuberculosis. Rev Soc Bras Med Trop. 2002 Sep-Oct;35(5):533-5.

10. Schulte C, Krebs B, Jelinek T, Nothdurft HD, von Sonnenburg F, Löscher T. Diagnostic significance of blood eosinophilia in returning travellers. Clin Infect Dis. 2002 Feb 1;34(3):407-11.

11. Makkar A, Rohtagi A, Goel A, Sharma SK, Garg S, Narayan S et.al.Study of Clinical Profile and Spontaneous Course of Eosinophilia. JK Science : Journal of Medical Education \& Research. 2005 Oct-Dec;7(4).

12. Brigten ML, Horak MG. Incidence and clinical significance of unsuspected eosinophilia discovered by automated WBC differential count. Lab Med. 1993;3:173-76.

13. Kobayashi S, Inokuma S, Setoguchi K, Kono H, Abe K. Incidence of peripheral blood eosinophilia and the threshold eosinophile count for indicating Hypereosinophilia- associated diseases. Allergy. 2002 Oct;57(10):950-6.
14. Limbos P. Etiology of eosinophilia in Europeans returning from Central Africa. Presented at XI international congress of internal medicine.1970:170-76.

15. Baldisserotto M, Conchin CF, Soares Mda G, Araujo MA, Kramer B. Ultrasound findings in children with toxocariasis: report on 18 cases. Pediatr Radiol. 1999 May;29(5):316-9.

16. Herry I, Philippe B, Hennequin C, Danel C, Lejeunne C, Meyer G. Acute life- threatening toxocaral tamponade. Chest. 1997 Dec;112:1692-3.

17. Yoko. A case report of toxocariasis with cardiac tamponade. Journal of Japan paediatric Society. 2004;108:1047-50.

18. Weller PF. Eosinophilic meningitis. Am J Med. 1993 Sep;95(3):250-3.

19. Boongird P, Phuapradit $P$, Siridej N, Chirachariyavej T, Chuahirun S, Vejjajiva A. Neurological manifestations of gnathostomiasis. J Neurol Sci. 1977 Mar;31(2):279-91.

20. Ying $M$, Xiaosu H, Wang B. A case of ectopic parasitism: Fasciola hepatica larvae burrow through a human brain and mimic cerebral aneurysm. Trans R Soc Trop Med Hyg. 2007 Oct;101(10):1051-2.

21. Zhou L, Luo L, You C, Wang B, Xu J, Liao L et.al. Multiple brain hemorrhages and hematomas associated with ectopic fascioliasis in brain and eye. Surg Neurol. 2008 May;69(5):516-21. 